\title{
Dexamethasone treatment inhibits VEGF production via suppression of STAT3 in a head and neck cancer cell line
}

\author{
SEON-HUI SHIM ${ }^{1,2}$, J. HUN HAH ${ }^{3}$, SO-YEON HWANG ${ }^{2}$, DAE SEOG HEO H, $^{1,4,5}$ and MYUNG-WHUN SUNG ${ }^{1,2,3,5}$ \\ ${ }^{1}$ Department of Molecular Tumor Biology, ${ }^{2}$ Cancer Research Institute, ${ }^{3}$ Department of Otorhinolaryngology, \\ Seoul National University College of Medicine; ${ }^{4}$ Department of Internal Medicine, Seoul National University Hospital; \\ ${ }^{5}$ Clinical Research Institute, Seoul National University College of Medicine, Seoul, Korea
}

Received October 26, 2009; Accepted December 18, 2009

DOI: $10.3892 /$ or_00000743

\begin{abstract}
Glucocorticoids (GCs) modulate the synthesis of many pro-inflammatory cytokines and influence multiple transduction pathways. GCs negatively or positively influence the transcription factors of their target genes. All of these transcription signals are closely connected to cancer survival or death. We investigated the action of dexamethasone (DEX) on head and neck cancer cell lines. When SNU-1041 and SNU-1076 were treated with DEX, the cell lines showed different patterns of responses. DEX inhibition of cell growth depended on concentration in SNU-1041, but not in SNU1076. Furthermore, DEX suppressed vascular endothelial growth factor (VEGF) secretion from SNU-1041, but not from SNU-1076. We explored the mechanism that explains these distinct differences. After DEX treatment, the differences of NF- $\mathrm{KB}$ (p65), glucocorticoid receptor and p-AKT were not observed between the cell lines. However, phospho-signal transducer and activator of transcription 3 (STAT3) decreased in SNU-1041 only. Moreover, STAT3 inhibition using si-RNA suppressed VEGF secretion. When STAT3 was overexpressed after DEX treatment, the level of VEGF in the culture media was restored. Taken together, we suggest that p-STAT3 can be a mediating factor which regulates VEGF secretion in the DEX treatment. Because the relationship between the three molecules DEX, STAT3 and VEGF is scarcely known, our findings clarified one of the signaling pathways of DEX, which is often used in clinical conditions.
\end{abstract}

\section{Introduction}

Dexamethasone (DEX), one of the synthetic glucocorticoids (GCs), is a widely used drug that suppresses symptoms of inflammation $(1,2)$. These drugs have been used in tumor

Correspondence to: Dr Myung-Whun Sung, Department of Otorhinolaryngology, Seoul National University College of Medicine, Seoul 110-799, Korea

E-mail: mwsung@snu.ac.kr

Key words: dexamethasone, signal transducer and activator of transcription 3, vascular endothelial growth factor, head and neck squamous cell carcinoma therapy for pro-apoptotic effects in lymphoid cells. However, the purpose of GCs in treatment of solid tumors is not to destroy malignant cells, but rather to alleviate symptoms caused by cancer therapy, such as acute toxicity, nausea and emesis (3).

GCs have diverse effects on different tissues and cells. GCs were reported to inhibit cell proliferation by causing the G1 phase of the cell cycle in lymphoid cells (4-7), but in solid tumors, the effect of DEX/GCs is still controversial. DEX prevented chemotherapy-induced apoptosis in some cell lines and xenograft models (8), while GCs exerted an anti-survival effect in small cell lung cancer and prostate cancer cells $(9,10)$.

In vivo administration of DEX can influence cancer cells as well as cells in surrounding tumor microenvironment. The relationship between GCs and angiogenesis draws attention. Many studies have reported that DEX treatment suppressed hypoxia-induced vascular endothelial growth factor (VEGF) mRNA and inhibited tumor angiogenesis in various cancer cells $(11,12)$. However, little is known about the molecular mechanisms of GCs on VEGF expression in head and neck cancer cells.

The effects of GCs are exerted through glucocorticoid receptors (GR). GR controls transcription by binding to GC response elements (GRE) in target gene's regulatory sequence or by modulating the activity of other transcription factors such as NF-кB, AP-1, SMAD3 and signal transducer and activator of transcription 3 (STAT3) (13). NF-кB is the most well-known transcription factor which regulates inflammation when DEX is treated; however, the STAT3 has not been fully studied after DEX treatment.

Signal transduction and activator of transcription 3 (STAT3) were associated with oncogenic transformation and a necessary requirement for malignant transformation. Many studies have shown that STAT3 inhibition facilitates decreasing tumor cell growth in vitro and in vivo by controlling genes including cyclin D1, Bcl-xL and VEGF in head and neck squamous cell carcinoma (HNSCC), NSCLC, glioma, breast cancer and prostate cancer (14). Moreover, STAT3 activity promotes in vivo angiogenesis by inducing the VEGF (15-17).

Until now, the molecular event of angiogenesis by DEX in head and neck cancer cells was not clearly identified. To understand the effects of GCs, we focused on the relationship among three molecules DEX, STAT3 and VEGF. By 
(A)

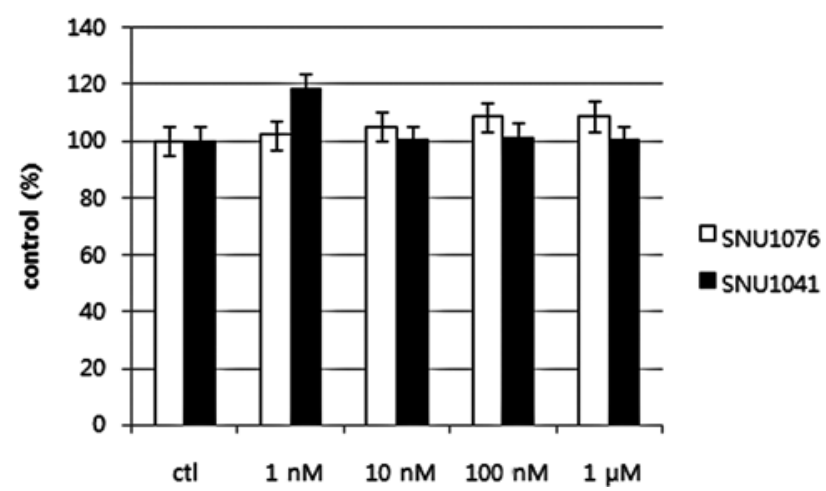

(B)

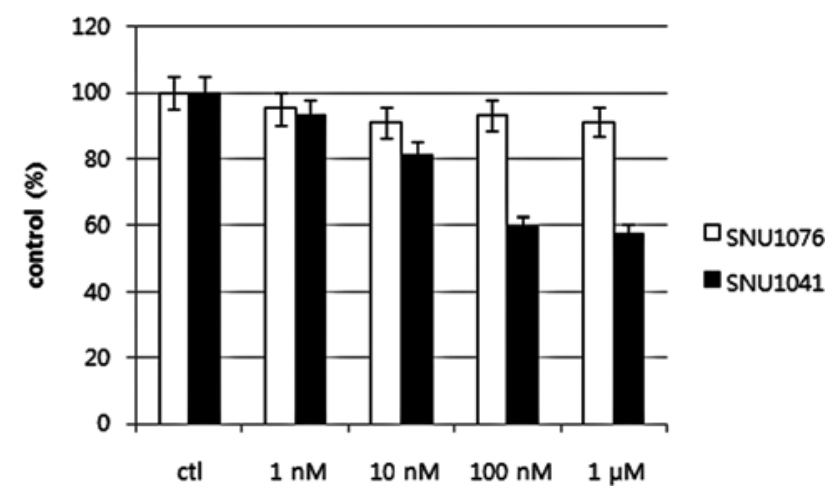

Figure 1. The cell proliferation was inhibited by DEX, only in SNU-1041. Black bars are for SNU-1041 and white bars for SNU-1076. The cells were treated with DEX in various doses ranging from 1 day (A) to 4 days (B). Error bars represent the standard error of the mean (P=0.00 Kruskal-Wallis method).

comparing two head and neck cancer cell lines in this study, we demonstrated that a decrease in STAT3 results in a reduction of VEGF by DEX.

\section{Materials and methods}

Cell lines and chemical. Human HNSCC cell lines SNU-1041 and SNU-1076 were purchased from the Korean Cell Line Bank (Seoul, Korea). Cell lines were cultured in RPMI-1640 medium with $10 \%$ fetal bovine serum and gentamycin (Gibco BRL, Grand Island, NY). Dexamethasone was obtained from Yuhan (Seoul, Korea).

Western blot analysis. Cultured cells were rinsed with PBS, suspended in lysis buffer (0.5\% NP40, $50 \mathrm{mM}$ Tris-Cl, $150 \mathrm{mM} \mathrm{NaCl}, 1 \mathrm{mM}$ dithiothreitol, $1 \%$ sodium deoxycholate, $0.1 \%$ SDS, 1 mM EDTA, $1 \mathrm{mM}$ phenylmethylsulfonyl fluoride, $0.1 \mathrm{M}$ aprotinin and $1 \mathrm{M}$ pepstatin $\mathrm{A}$ ). The cell lysates were then centrifuged at 13,000 rpm for $20 \mathrm{~min}$ at $4^{\circ} \mathrm{C}$. An appropriate amount of each supernatant (determined by protein assay) was mixed with $4 \mathrm{X}$ sample loading buffer and denatured for $10 \mathrm{~min}$ at $70^{\circ} \mathrm{C}$. The denatured protein samples were fractionated on $4-12 \% \mathrm{NuPAGE}^{\mathrm{TM}}$ Bis-Tris gels (Invitrogen, Carlsbad, CA), transferred onto nitrocellulose membranes (Schleicher \& Schuell, Dachen, Germany) and incubated with Tris-buffered saline containing $0.1 \%$ Tween-20 (TBS-T) and 5\% non-fat dry milk. The membranes were then incubated with p-AKT, AKT, p-ERK, ERK, GR, $\alpha$-tubulin (Santa Cruz Biotechnology, Santa Cruz, CA), p-STAT3, STAT3 (Cell Signaling, Danvers, MA) antibody. Experiments were repeated at least three times.

Cell proliferation assay. Exponentially growing cells were trypsinized, seeded at a specific density $\left(10^{4}\right.$ cells/well in a flat-form 96-well plate) and then grown for $24 \mathrm{~h}$. After $24 \mathrm{~h}$, fresh medium with various concentrations of chemicals was added. Following a 5-day incubation, an MTT assay (Amresco, Solon, $\mathrm{OH}$ ) was performed. Twenty microliters of MTT solution ( $2 \mathrm{mg} / \mathrm{ml}$ in deionized water) was added to each well, and then incubated for $4 \mathrm{~h}$. After removal of the supernatant, $120 \mu 1$ of DMSO was added and the pellet was resuspended for 30 min with vigorous shaking. The optical density was determined at a wavelength of $564 \mathrm{~nm}$ using an ELISA reader (Molecular Devices, Sunnyvale, CA).

Wild-type STAT3 and small-interfering RNA transfection. Wild-type STAT3 was kindly provided by Dr Jung Weon Lee, Seoul National University (18). ON-TARGET ${ }^{\mathrm{TM}}$ si-RNA was purchased from Dharmacon (Chicago, IL). After the cells had grown for $24 \mathrm{~h}, 4 \mu \mathrm{l}$ of Lipofectamin 2000 (Invitrogen) and $200 \mathrm{nM} / 1$ small-interfering RNA or $1 \mu \mathrm{l}$ of plasmid solution were incubated at room temperature for $15 \mathrm{~min}$ with RPMI-1640 media. The cells were then treated with the transfection complexes for $24 \mathrm{~h}$. After $24 \mathrm{~h}$, the media were removed and fresh media. The cells were incubated for 48 or $72 \mathrm{~h}$.

VEGF quantification. For quantitative determination of human VEGF in cell culture supernatants, the immunoassay kit (Invitrogen) was used according to the manufacturer's instructions.

Statistical analysis. Data shown in the graphs represent the mean value \pm standard deviation (SD) of three independent experiments. Statistical analysis was performed using SPSS software (SPSS Inc., Chicago, IL).

\section{Results}

The growth inhibition by DEX. To evaluate the response of DEX, growth inhibition was tested in the head and neck cancer cell lines SNU-1041 and SNU-1076. Fig. 1 shows the cell growth inhibition in SNU-1041 in a dose-dependent manner, but no effect was seen in SNU-1076.

The secretion of VEGF by DEX. We tested whether different effects were shown on VEGF level between two cell lines as well. The pattern of response on DEX was similar to Fig. 1. VEGF secretion decreased significantly in SNU-1041, but there was no effect seen in SNU-1076 (Fig. 2).

The different expression of transcription factors. To understand the mechanism that explains this clear difference between the 
(A)

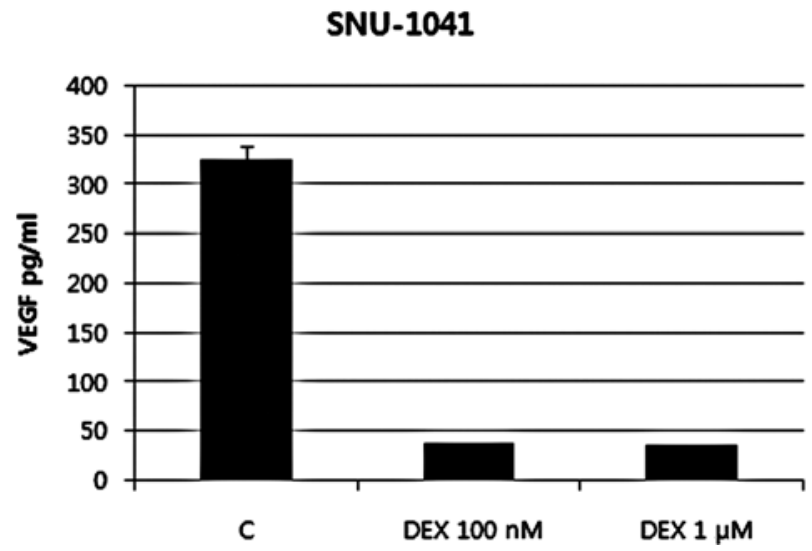

(B)

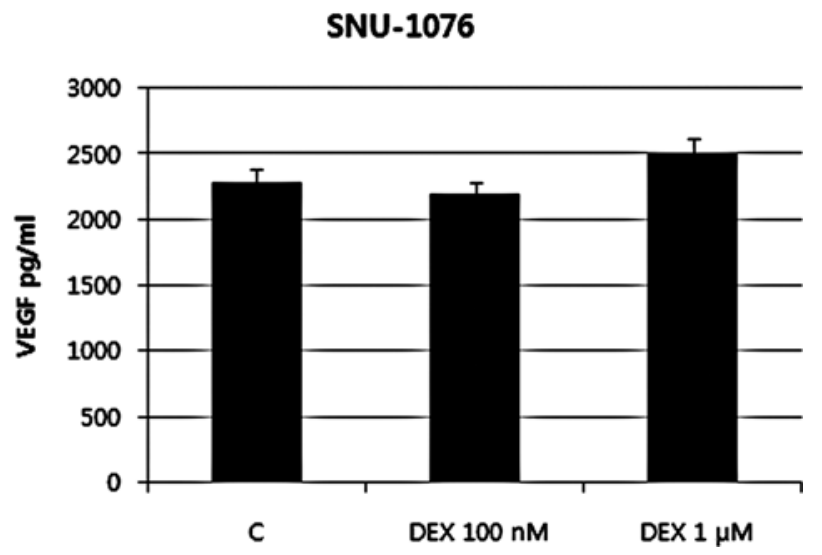

Figure 2. VEGF secretion in culture medium decreased in SNU-1041 but no change was seen in SNU-1076 (A, SNU-1041; B, SNU-1076). VEGF was measured at $48 \mathrm{~h}$ after DEX treatment at doses of $100 \mathrm{nM}$ and $1 \mu \mathrm{M}(\mathrm{P}<0.05 \mathrm{Kruskal}-$ Wallis method $)$.

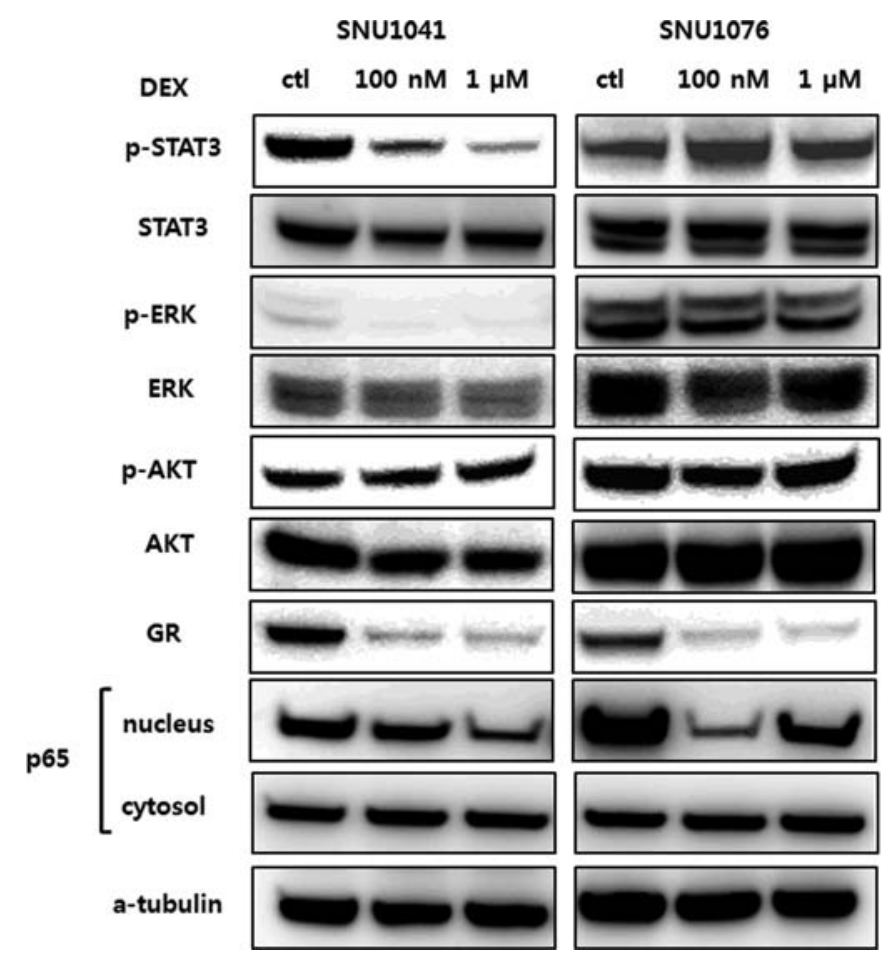

Figure 3. To evaluate the expression of protein molecules in signaling pathways, cells were treated with DEX for $48 \mathrm{~h}$. Phospho-STAT3 was found to be suppressed by DEX in SNU-1041, but there was no effect in SNU-1076. GR and p65 decreased in both cell lines and no effect was shown in AKT. Experiments were repeated at least three times.

two cell lines, we investigated signaling molecules related to glucocorticoids. We screened the change of various signaling molecules by different concentration of DEX on each cell line. Among them, phospho-STAT3 in SNU-1041 was found to decrease after DEX treatment, but not in SNU-1076. The response of p-ERK was similar to that of STAT3, but the basal level was very weak. Thus we hypothesized that STAT3 could be a signaling molecule that leads to the different pattern of growth and VEGF secretion. The other molecules tested did not show any difference between the two cell lines. GR, NF-кB and p-AKT responded similarly in both cell lines.

Change of VEGF secretion by modulating STAT3 activity. To verify that STAT3 is the signaling molecule which determines the different responses on the treatment of DEX, we investigated the level of VEGF secretion when STAT3 was down-regulated by si-RNA of STAT3. Fig. 4 shows a significant decrease of VEGF in culture medium when the activity of STAT3 was suppressed.

Restoring VEGF secretion by STAT3 overexpression after $D E X$. To confirm our hypothesis, STAT3 was overexpressed by transfecting wild-type STAT3 into SNU-1041 (Fig. 5A). Cells transfected with wild-type STAT3 showed a significant increase of VEGF secretion after DEX treatment (Fig. 5B). Taken together, STAT3 seems to be one of the important molecules mediating the signaling pathway of VEGF reduction by DEX treatment in SNU-1041.

\section{Discussion}

A large number of studies with tumor cell lines and patient samples have provided evidence for the role of constitutive STAT3 activity in human tumors. In our previous study inhibition of STAT3 led to growth arrest in head and neck cancer cell lines, and many other studies have supported that blocking STAT3 can be an important element in cancer therapy (14). In this study, two different cell lines originated from head and neck cancer patients were treated with DEX. STAT3 inhibition by DEX was observed in SNU-1041, resulting in cell growth arrest. However, neither change of STAT3 nor growth arrest was observed in the SNU-1076 cell line (Fig. 1). This interesting finding and a direct proportional relationship between STAT3 and cell growth from our previous observation suggested that STAT3 would be an important target when suppressing tumor cells (19).

Many studies have defined an essential role of activated STAT3 in tumor angiogenesis in a wide range of cancers 
(A)

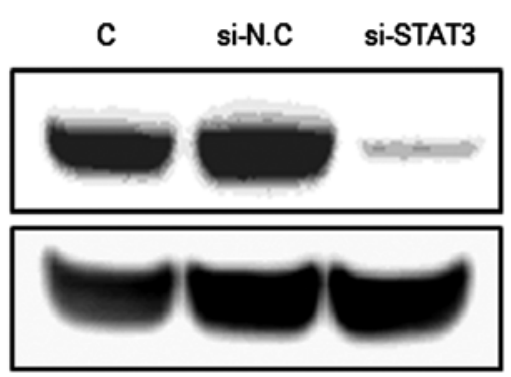

STAT3

a-tubulin

Figure 4. Down-regulation of STAT3 using si-RNA caused suppression of VEGF secretion in culture medium. STAT3 was repressed by si-STAT3 (A). VEGF secretion was measured after si-STAT3 transfection in SNU-1041 (B) $\left({ }^{*} \mathrm{P}=0.02\right)$ (si-n.c:si-normal control)

(A)

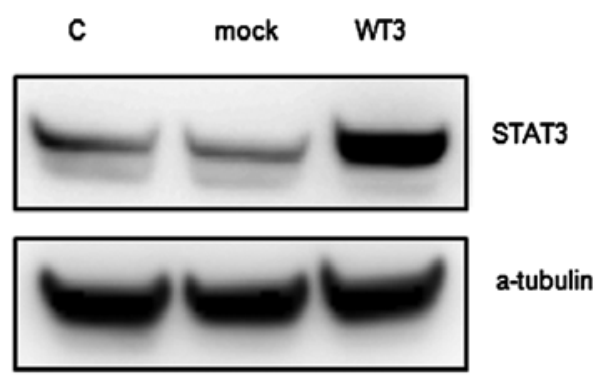

Figure 5. Wild-type STAT3 overexpression led to increased VEGF secretion under DEX treatment. Western blot analysis shows overexpression of STAT3 after transfection of wild-type STAT3 expression vector (wt3) (A) The bar graph represents VEGF secretion. VEGF secretion was restored after wild-type STAT3 (wt3) trasnfection (B). DEX was pre-treated for $8 \mathrm{~h}$ before STAT3 transfection. Mock denotes empty vector $\left({ }^{*} \mathrm{P}=0.00\right)$.

including breast, gastric, pancreatic and head and neck cancers (14). Multiple studies on the link between STAT3 and VEGF suggested that STAT3 influences the regulation of VEGF as well as a direct transcription factor of VEGF promoter $(20,21)$. Cytokines participate in VEGF-mediated angiogenesis via diverse signaling pathways such as IGF-1, TGF-ß (22). Also IL-6 has been reported as a stimulator of STAT3 (21). Accumulating data suggest that STAT3 should be a pivotal factor of angiogenesis (21). However, for glucocorticoids, it was still uncertain that STAT3 is a mediator between glucocorticoids and VEGF.

GR expression levels are down-regulated in most cell types in response to GCs but appear to be up-regulated in lymphoid

(B)

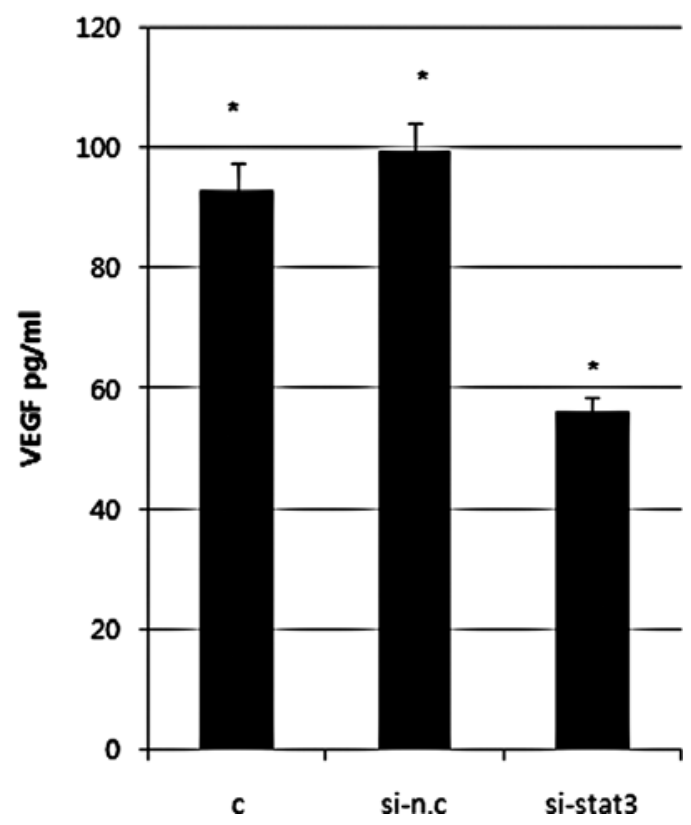

(B)

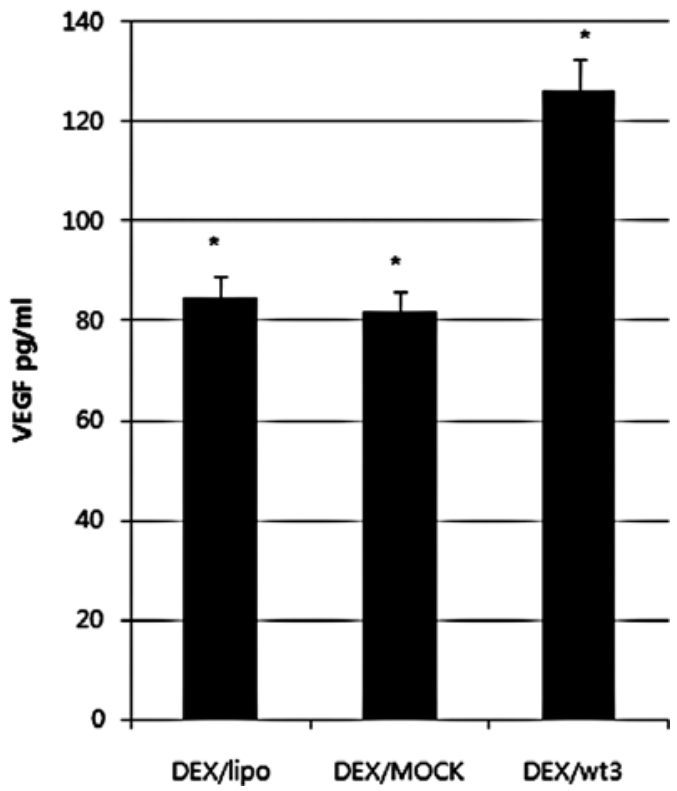

cells (23). The direct positive transcriptional regulation occurs via the binding of the GR to glucocorticoid-response elements (GRE). The indirect regulation is mediated via crosstalk with other transcription factors including AP-1, NF$\kappa \mathrm{B}, \mathrm{STAT}, \mathrm{SMAD}$. The interaction between GR and transcription factors is likely to repress the activity of partner transcription factor and their target genes (13). After stimulation of GCs, GR responds in various ways. One study showed a decrease in GR expression in HeLa and A549 as seen in our data (Fig. 3), while another reported an increasing GR expression after DEX treatment $(8,24)$. An extensive study using microarray techniques was conducted in order to identify GC-regulated genes responsible for the induction of apoptosis, 
but the results are conflicting and inconclusive (6). This implies that genes might be regulated in different cell systems and experimental conditions. Thus it is uncertain yet what factors determine the direction or response between GR and transcription factors after DEX treatment.

Surgical resection is one of the most important treatments for head and neck cancer patients. Surgery causes inevitable inflammatory response in tumor microenvironment; therefore, GCs might be beneficial in reducing acute phase inflammatory reaction, even though GCs may possibly suppress immune response against cancer. One study has shown beneficial results of DEX treatment for the host after resection of pancreatic cancer (25). DEX was found to reduce metastasis and size of local tumor recurrence (25). Therefore, to maximize the efficacy of cancer therapy, more specific understanding of the cancer cells themselves and the tumor microenvironment is necessary, because the effect of DEX on solid tumor can be still double-edged.

In this study, we clearly demonstrated that DEX could reduce VEGF secretion in some head and neck cancer cells via STAT3. In conclusion, we suggest that active STAT3 is a mediating signal of VEGF reduction caused by DEX in head and neck cancer cells. Because DEX is often used in clinical conditions, clear understanding of the role of STAT3 in DEX treatment is important in clinical decision-making. Furthermore, these findings may imply that STAT3 could be a key molecule in modulating the inflammatory response in tumor microenvironment.

\section{Acknowledgements}

This study was supported by grant no. 04-2008-091-0 from the SNUH Research Fund and supported by a grant of the Korea Healthcare Technology R\&D Project, Ministry for Health, Welfare \& Family Affairs, Republic of Korea (A060141).

\section{References}

1. Kofler R: The molecular basis of glucocorticoid-induced apoptosis of lymphoblastic leukemia cells. Histochem Cell Biol 114: 1-7, 2000.

2. Planey SL and Litwack G: Glucocorticoid-induced apoptosis in lymphocytes. Biochem Biophys Res Commun 279: 307-312, 2000.

3. Evans-Storms RB and Cidlowski JA: Delineation of an antiapoptotic action of glucocorticoids in hepatoma cells: the role of nuclear factor-kB. Endocrinology 141: 1854-1862, 2000

4. Sanchez I, Goya L, Vallerga AK and Firestone GL: Glucocorticoids reversibly arrest rat hepatoma-cell growth by inducing an early G(1) block in cell-cycle progression. Cell Growth Differ 4: 215-225, 1993.

5. Wang Z and Garabedian MJ: Modulation of glucocorticoid receptor transcriptional activation, phosphorylation, and growth inhibition by p27(Kip1). J Biol Chem 278: 5089750901, 2003

6. Schmidt S, Rainer J, Ploner C, Presul E, Riml S and Kofler R: Glucocorticoid-induced apoptosis and glucocorticoid resistance: molecular mechanisms and clinical relevance. Cell Death Differ 11: S45-S55, 2004.
7. Harmon JM, Norman MR, Fowlkes BJ and Thompson EB: Dexamethasone induces irreversible G1 arrest and death of a human lymphoid-cell line. J Cell Physiol 98: 267-278, 1979.

8. Herr I, Ucur E, Herzer K, Okouoyo S, Ridder R, Krammer PH, von Knebel Doeberitz M and Debatin KM: Glucocorticoid cotreatment induces apoptosis resistance toward cancer therapy in carcinomas. Cancer Res 63: 3112-3120, 2003.

9. Sommer P, Le Rouzic P, Gillingham H, Berry A, Kayahara M, Huynh T, White A and Ray DW: Glucocorticoid receptor overexpression exerts an antisurvival effect on human small cell lung cancer cells. Oncogene 26: 7111-7121, 2007.

10. Yemelyanov A, Czwornog J, Chebotaev D, Karseladze A, Kulevitch E, Yang X and Budunova I: Tumor suppressor activity of glucocorticoid receptor in the prostate. Oncogene 26 : 1885-1896, 2007

11. Yano A, Fujii Y, Iwai A, Kageyama Y and Kihara K: Glucocorticoids suppress tumor angiogenesis and in vivo growth of prostate cancer cells. Clin Cancer Res 12: 3003-3009, 2006.

12. Wilson C, Scullin P, Worthington J, Seaton A, Maxwell P, O'Rourke D, Johnston PG, McKeown SR, Wilson RH, O'Sullivan JM and Waugh DJ: Dexamethasone potentiates the antiangiogenic activity of docetaxel in castration-resistant prostate cancer. Br J Cancer 99: 2054-2064, 2008.

13. Necela BM and Cidlowski JA: Mechanisms of glucocorticoid receptor action in noninflammatory and inflammatory cells. Proc Am Thorac Soc 1: 239-246, 2008.

14. $\mathrm{Yu} \mathrm{H}$ and Jove R: New molecular targets come of the age. Nat Rev Cancer 4: 97-105, 2004

15. Niu G, Wright KL, Huang M, Song L, Haura E, Turkson J, Zhang S, Wang T, Sinibaldi D, Coppola D, Heller R, Ellis LM, Karras J, Bromberg J, Pardoll D, Jove R and Yu H: Constitutive STAT3 activity upregulates VEGF expression and tumor angiogenesis. Oncogene 21: 2000-2008, 2002.

16. Xie TX, Huang FJ, Aldape KD, Kang SH, Liu M, Gershenwald JE, Xie K, Sawaya R and Huang S: Activation of STAT3 in human melanoma promotes brain metastasis. Cancer Res 66: 3188-3196, 2006.

17. Xu Q, Briggs J, Park S, Niu G, Kortylewski M, Zhang S, Gritsko T, Turkson J, Kay H, Semenza GL, Cheng JQ, Jove R and Yu H: Targeting STAT3 blocks both HIF-1 and VEGF expression induced by multiple oncogenic growth signaling pathways. Oncogene 24: 5552-5560, 2005.

18. Jung JE, Lee HG, Cho IH, Chung DH, Yoon SH, Yang YM, Lee JW, Choi S, Park JW, Ye SK and Chung MH: STAT3 is a potential modulator of HIF-1-mediated VEGF expression in human renal carcinoma cells. FASEB J 19: 1296-1298, 2005.

19. Shim SH, Sung MW, Park SW and Heo DS: Absence of STAT1 disturbs the anticancer effect induced by STAT3 inhibition in head and neck carcinoma cell lines. Int J Mol Med 23: 805-810, 2009.

20. Fidler IJ and Ellis LM: The implications of angiogenesis for the biology and therapy of cancer metastasis. Cell 79: 185-188, 1994.

21. Chen Z and Han ZC: STAT3: a critical transcription activator in angiogenesis. Med Res Rev 28: 185-200, 2008.

22. Folkman J: Angiogenesis in cancer, vascular, rheumatoid and other disease. Nat Med 1: 27-31, 1995.

23. Burnstein KL, Bellingham DL, Jewell CM, Powell-Oliver FE and Cidlowski JA: Autoregulation of glucocorticoid receptor gene expression. Steroids 56: 52-58, 1991

24. Matthews L, Berry A, Tersigni M, D'Acquisto F, Ianaro A and Ray D: Thiazolidinediones are partial agonists for the glucocorticoid receptor. Endocrinology 150: 75-86, 2009.

25. Egberts JH, Schniewind B, Patzold M, Kettler B, Tepel J Kalthoff $\mathrm{H}$ and Trauzold $\mathrm{A}$ : Dexamethasone reduces tumor recurrence and metastasis after pancreatic tumor resection in SCID mice. Cancer Biol Ther 7: 1044-1050, 2008. 